\title{
Compared to American system, which future for regulatory affairs of medical devices in Tunisia
}

\section{Mahmoud Zarati ${ }^{1}$, Ines Fradi ${ }^{2}$, Chema Drira ${ }^{3}$}

${ }^{1}$ Intern at National Center for Bone Marrow Transplantation, Tunisia, ${ }^{2}$ Director of Pharmacy and medicines direction, Tunisia, ${ }^{3}$ Dr at National Center for Bone Marrow Transplantation, Tunisia

As a leader of regulatory science in the world, FDA has developed an efficient regulatory system of medical devices. In Tunisia and around the world, it seems to be challenging to establish a real efficient regulation system. The aim of this research is to study and analyse the american regulation of medical devices to propose recommendations to newly set systems in Tunisia and other countries around the world. 